EUROPEAN ORGANIZATION FOR NUCLEAR RESEARCH

European Laboratory for Particle Physics

\author{
LHC Project Report 55
}

\title{
Field Errors Decay and "Snap-Back" in LHC Model Dipoles
}

\author{
L. Bottura, L. Walckiers, R. Wolf
}

\begin{abstract}
The magnetic field in accelerator magnets decays when the current is kept constant during the particles injection phase, and returns quickly (snaps back) to the original values as soon as ramping is restarted. Here we show results of measurements of the decay of the field errors in $10 \mathrm{~m}$ long LHC model dipole magnets. In accordance with previous findings, precycles and stops at intermediate current levels influence the decay. We discuss a possible mechanism causing the decay and snap-back, based on the internal field change in the cable.
\end{abstract}

*LHC Division

Paper presented at 1996 Applied superconductivity Conference, Pittsburgh, Pa, August 25-30, 1996

Admnistrative Secretariat

LHC Division

CERN

CH - 1211 Geneva 23

Switzerland

18 September 1996 


\title{
Field Errors Decay and "Snap-Back" in LHC Model Dipoles
}

\author{
L. Bottura, L. Walckiers, R. Wolf \\ CERN, CH-1211 Geneva, Switzerland
}

\begin{tabular}{|c|c|c|}
\hline $\begin{array}{l}\text { Abstract- } \\
\text { when the } \\
\text { phase, an } \\
\text { as soon as } \\
\text { urements } \\
\text { model di } \\
\text { precycles } \\
\text { decay. W } \\
\text { snap-back }\end{array}$ & $\begin{array}{l}\text { he magnetic field in accelerator } \\
\text { rrent is kept constant during the par } \\
\text { returns quickly (snaps back) to the } \\
\text { amping is restarted. Here we show } r \\
\text { the decay of the field errors in } 10 \\
\text { le magnets. In accordance with pre } \\
\text { d stops at intermediate current leve } \\
\text { liscuss a possible mechanism causing } \\
\text { based on the internal field change in }\end{array}$ & $\begin{array}{l}\text { lets d } \\
\text { es inje } \\
\text { inal v } \\
\text { ts of } n \\
\text { long } \\
\text { find } \\
\text { fluenc } \\
\text { decay } \\
\text { cable. }\end{array}$ \\
\hline & $\begin{array}{c}\text { TABLE I } \\
\text { LIST OF SYMBOLS }\end{array}$ & \\
\hline Parameter & Description & $\overline{U n i t(S I)}$ \\
\hline$z=x+i y$ & complex variable & \\
\hline $\boldsymbol{B}=B_{v}+i B_{x}$ & Magnetic field & $(\mathrm{T})$ \\
\hline$B_{n}, A_{n}$ & Normal and skew harmonic coefficients & ( $\mathrm{T})$ \\
\hline $\begin{array}{l}b_{n}^{n}, a_{n} \\
\mathrm{R}_{\mathrm{ref}}\end{array}$ & $\begin{array}{l}\text { Normalised harmonic coefficients } \\
\text { reference radius }\end{array}$ & (m) \\
\hline$M / /, M \perp$ & $\begin{array}{l}\text { Magnetization parallel and perpendicular to } \\
\text { the field of the magnet }\end{array}$ & (T) \\
\hline$\Delta B$ & change of magnetization & (T) \\
\hline$\Delta M$ & change of field & (T) \\
\hline$|\Delta J|$ & $\begin{array}{l}\text { change of transport current density in a } \\
\text { strand, absolute value }\end{array}$ & $\left(\mathrm{A} / \mathrm{m}^{2}\right)$ \\
\hline$|\Delta J|_{\text {avg }}$ & average $|\Delta \mathrm{J}|$ of all strands in a cable. & $\left(\mathrm{A} / \mathrm{m}^{2}\right)$ \\
\hline$|\Delta B|_{\text {avg }}$ & $\begin{array}{l}\text { average over half a cable twist of the } \\
\text { change of the field component parallel to } \\
\mathrm{M} / / \text {. }\end{array}$ & $(\mathrm{T})$ \\
\hline$J c$ & critical current density of strand & $\left(\mathrm{A} / \mathrm{m}^{2}\right)$ \\
\hline$d$ & filament diameter & (m) \\
\hline$L_{p}$ & Cable twist pitch & (m) \\
\hline$N$ & number of strands in a cable & $(\mathrm{m})$ \\
\hline$\lambda$ & cross-section filaments/cross-section cable & \\
\hline$\eta$ & cross-section strands/cross-section cable & \\
\hline
\end{tabular}

\section{INTRODUCTION}

Superconducting magnets wound with cables exhibit a long-term drift of the magnetic field during plateaux of current, with typical time constant in the order of several minutes to several hours. The drift is recovered completely when ramping is restarted: the magnetic field snaps back to its original value at the beginning of the current plateau. The order of magnitude of these field errors is comparable to the expected geometric systematic and random errors at injection in the Large Hadron Collider (LHC) [1,2]. Therefore this error component will influence significantly the LHC operation and reproducibility at beam injection and start of energy ramp. This is the main motivation for this work.

Early evidence of long-term drift of the magnetic field at constant operating current deduced from chromaticity changes in Tevatron [3] was supported by later direct measurements on single dipoles $[4,5]$. These measurements suggested that the field drift is associated with a change in cable magnetization[4] rather than with cable eddy currents with long time constant. Hanft et al. [5] showed that powering cycles performed before the measurement increased the field decay. In particular, higher currents and precycle repetition gave stronger decays. Similar results were obtained on prototypes SSC dipoles[6], and on the HERA dipoles[7]. A tentative

Manuscript received August 25, 1996. explanation based on flux creep could not be substantially confirmed, owing on one side to large disparity between the expected creep rate and the much larger field drift measured, and on the other hand to the dependence on the powering history mentioned above.

We will refer in the discussion to the harmonic components of the 2-D complex expansion of the field in the magnet cross section, defined as:

$$
\mathbf{B}=\sum_{n=1}^{\infty}\left(B_{n}+i A_{n}\right)\left(\frac{z}{R_{r e f}}\right)^{n-1}=B_{1} \sum_{n=1}^{\infty} \frac{\left(b_{n}+i a_{n}\right)}{10^{4}}\left(\frac{z}{R_{r e f}}\right)^{n-1} .
$$

We will concentrate in particular on the first allowed harmonics of the main dipole field $B_{1}$, namely the normal sextupole $b_{3}\left(\right.$ or $\left.B_{3}\right)$ and decapole $b_{5}$ (or $\left.B_{5}\right)$, most likely to cause systematic effects in LHC. These components are quoted at the reference position $R_{r e f}=10 \mathrm{~mm}$.

\section{EXPERIMENTAL RESULTS}

We report results from two $10 \mathrm{~m}$ long dipole models, MTP1A3 and MTP1N2. They have manufacturing scale close to the main dipoles of the LHC. The bore diameter of these models is $50 \mathrm{~mm}$, as contrasted to the reference LHC aperture of $56 \mathrm{~mm}[1]$. For all measurements the magnets were quenched and cycled to a flat-top at 12 to $13 \mathrm{kA}(8$ to $8.5 \mathrm{~T})$, followed by ramp-down to minimum current $(550 \mathrm{~A}$, around $0.4 \mathrm{~T}$ ) before bringing the magnet to the measurement current plateau at injection level $(880 \mathrm{~A}$, around $0.6 \mathrm{~T})$. Due to magnet performance limitations the precycle was not identical for the two models. Therefore the results from the two magnets cannot be directly compared, but are rather intended for relative comparisons (using results from the same magnet). The field was measured with a $750 \mathrm{~mm}$ long rotating coil in the centre of the straight section. Local measurements of field and field periodicity were taken with an array of 7 short (25 $\mathrm{mm}$ ) rotating coils.

\section{A. Influence of Precycle Flat-top Parameters}

Fig. 1 shows the influence of the precycle maximum (flattop) current and of the flat-top duration on the sextupole decay in the MTP1A3 model. These results confirm previous findings discussed earlier, namely that precycling at higher currents or for longer times increases the decay of the field. A detailed inspection of the curves shows however a significant difference in the effect of a higher flat-top current and of a longer flat-top time. While the latter only affects the decay amplitude, the former affects both decay amplitude and initial value. We evidence the correlation of allowed harmonics changes in Fig. 2, where we have plotted normal sextupole and decapole decays for the same cases shown in Fig. 1. Note how a strong correlation is found for high precycle current (for different flat-top durations), but also how this correlation breaks-down at low precycle currents. 

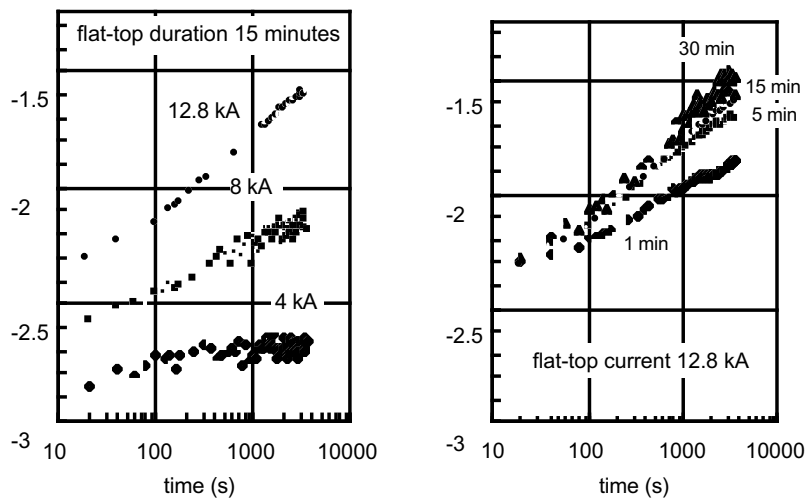

Fig. 1. Normal sextupole drift at constant current $(880 \mathrm{~A}, 0.6 \mathrm{~T})$ in the MTP1A3 model dipole, as a function of the maximum current reached in the precycle (left) and of the duration of the maximum current flat-top in the precycle (right)
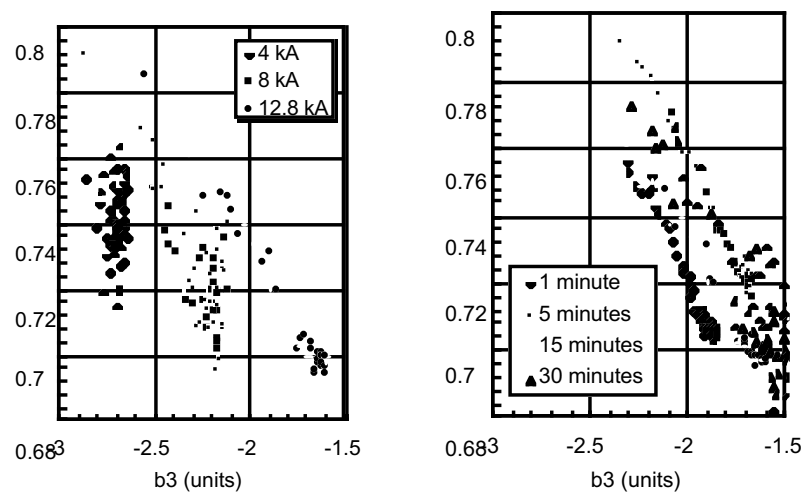

Fig. 2. Correlation between the changes of normal sextupole and decapole at constant current $(880 \mathrm{~A}, 0.6 \mathrm{~T})$ in the MTP1A3 model dipole, as a function of the maximum current reached in the precycle (left) and of the duration of the maximum current flat-top in the precycle (right). Same conditions as in Fig. 1.

\section{B. Influence of Precycle Intermediate Stops}

We have measured the effect of intermediate stops in the cycle at low current, before reaching the injection current. Similarly to results reported on SSC magnets [6], we have found that any stop close to the injection level decreases the subsequent field decay at injection. The gain in terms of a reduced $b_{3}$ decay is approximately independent on the current where the previous stops have taken place, and increases when the total waiting time at low field before injection is increased.

\section{Snap-back}

As the ramping is restarted at the end of the constant current injection phase, the field snaps back to its original value. We have reported a typical curve measured on the MTP1N2 model in Fig. 3 (crosses). Measuring the details of the snapback phase we have found that for the LHC dipoles the initial field value is recovered within 20 to $30 \mathrm{~A}$ current increase (15 to $20 \mathrm{mT}$ field increase). The time scale of the field recovery is inversely proportional to the ramping speed [2]. This demonstrates that snap-back is a $\Delta \mathrm{B}$ (field change) rather than

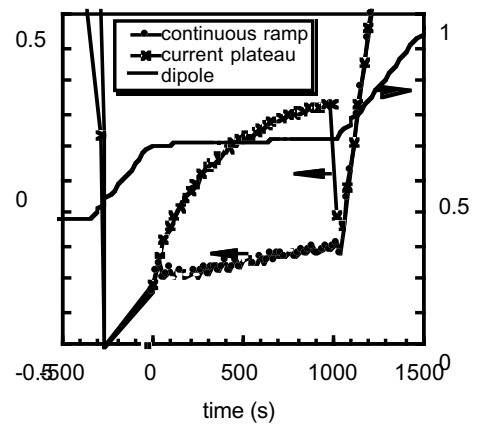

Fig. 3. Normal sextupole decay during the injection plateau ( 0 to $1000 \mathrm{~s})$ and snap-back as ramping is restarted (at $1000 \mathrm{~s}$ ). The results for a constant current injection plateau at $890 \mathrm{~A}$ and for an artificial small ramp (20 A in $1000 \mathrm{~s}$ ) are compared. Also reported the measured dipole field in the second case.

$\mathrm{a} \mathrm{dB} / \mathrm{dt}$ (change rate) process. This is of extreme importance for the LHC operation, as a slower ramp will allow a precise correction algorithm to be implemented.

To give further evidence of the fact that the snap-back is associated to the field change $\Delta \mathrm{B}$, we have performed a measurements during an injection plateau with an artificial and small continuous current change of $20 \mathrm{~A}$ in 1000 s, i.e. of the same order of magnitude of the current change necessary for the snap-back to take place. We would expect to see no abrupt snap-back, as by the end of the gently sloped injection phase we have produced the necessary $\Delta \mathrm{B}$ to cancel it (see the dipole waveform, the continuous line in Fig. 3). Indeed, the results of Fig. 3 (circles) show that this is the case. In fact, examining the fine structure of the curve, the normal sextupole goes through a series of mini-snap-backs, which correspond to the discrete steps of $1 \mathrm{~A}$ taken by the power supply to simulate the $20 \mathrm{~mA} / \mathrm{s}$ slope.

\section{Current Distribution and Operating Temperature}

As already known [8-10] the local field exhibits variations on the length scale of a cable twist pitch. These local variations are most probably due to the current distribution in the strands of each cable. Using an array of short coils it is possible to measure simultaneously both the local value of the field and the average field over a cable twist pitch. From the amplitude of the periodic field variation we can infer the current imbalance. The average, on the other hand, should be insensitive to current distribution. Measurements have been performed with such a system on the MTP1N2 model, taking as additional parameter the operating temperature during the injection plateau. Fig. 4 shows a summary of the results in term of $\mathrm{B}_{3}$ (non-normalised sextupole) peak-to-peak amplitude and average value for three cases:

\section{1. constant temperature of $1.8 \mathrm{~K}$}

2. temperature increase during injection from 1.8 to $4.3 \mathrm{~K}$

3. temperature decrease during injection from 2 to $1.6 \mathrm{~K}$

Both current distribution (amplitude) and average $\mathrm{B}_{3}$ change during injection, as reported earlier [10]. At the restart of the ramp, however, the current distribution does not show any sudden changes, while the usual average snap-back pic- 


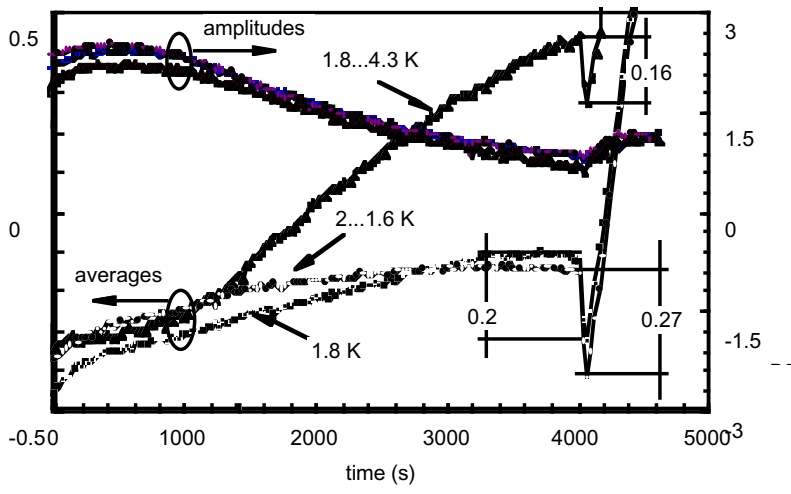

Fig. 4. Results of local field measurements during injection plateau in the MTP1N2 model. The normal sextupole $B_{3}$ average value and amplitude of longitudinal periodicity are reported for constant (squares), increasing (triangles) and decreasing (circles) temperature conditions during injection.

ture is obtained. Therefore the snap-back is not associated to a change of current distribution in the cable at the restart of the ramp. Furthermore, the snap-back is different depending on the temperature. At $1.6 \mathrm{~K}$ a snap-back amplitude of 0.27 Gauss@10 mm is measured, to be compared to about 0.16 Gauss@10 mm measured at $4.3 \mathrm{~K}$. The ratio of these two (1.6) is approximately the same as the ratio of the contributions of the cable magnetization to the normal sextupole measured at approximately the same temperatures. We have here a further evidence that decay and snap-back are associated with a de-magnetized volume, that becomes magnetized again as ramping is restarted. The amount of magnetization recovered is proportional in this case to the critical current density through the proper geometric factor for the sextupole.

\section{THE DECAY MECHANISM}

We believe that the decay of the field error is caused by the change of the initial filament magnetization $M$ due to the current redistribution among the strands of the cable[10]. The current redistribution changes the internal field in the cable and, as is shown below, reduces $M$. The idea that current redistribution reduces $M$ is not new [11], although the mechanism proposed earlier seems unlikely. We identify two mechanisms associated with current redistribution that lead to a strand magnetization decrease: strand self field effect[12] and external field change (cumulative effect from all strands in the cable and the neighbouring turns)

The magnetization change due to the self field for one strand is always negative $\Delta M=-\frac{2 \mu_{0}}{3} d|\Delta J|$, because the transport current change tends to wipe-out the magnetization

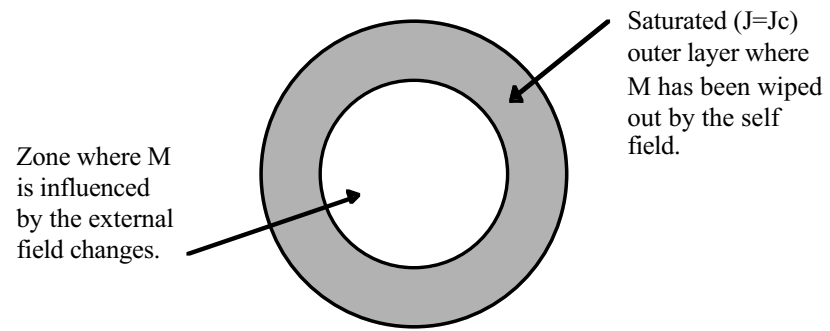

Fig.5. Sketch of the regions of a strand where the magnetization(M) changes are due to self field and to external field. of the outer shell of the strand (see Fig. 5). As each strand is twisted in the cable, the average magnetization change over the cable volume can be obtained from the average change of strand transport current density as:

$\Delta M_{\text {self }}=-\frac{2 \mu_{0}}{3} \eta d|\Delta J|_{\text {avg }}$.

In addition, each strand sees a changing external field which rotates along the cable with period equal to the cable twist pitch $L_{p}$, similar to what is measured in the aperture [810]. The direction of the field change is therefore not necessarily parallel to the initial magnetic moment $M$ of the filament. the oscillating external field increases over approximately one half of the twist pitch and decreases over the other half. For a field increase the magnetization remains on the same branch of the hysteresis loop and changes little, as the injection plateau was reached increasing the field (see Fig. 6). On the other hand, for a field decrease a new penetration layer is formed with reversed shielding currents, causing a large change of the magnetization. In summary, the magnetization is lowered over approximately half a cable pitch, while it changes little over the other half (see Fig.6). Besides a magnetization component perpendicular to the original one is generated. This perpendicular component does not give a significant contribution to the field error integral. For small field changes $\left(<<J_{c} d\right)$ the change of the original magnetization is estimated as:

$$
\Delta M_{e x t} \approx-\frac{\lambda}{2}|\Delta B|_{\text {avg }}\left[1-\frac{|\Delta J|_{\text {avg }}}{J_{c}}\right] \text {. }
$$

In conclusions, on the average the magnetization always decreases during a current plateau. For small current changes compared to the critical current, the total change of $M$ is:
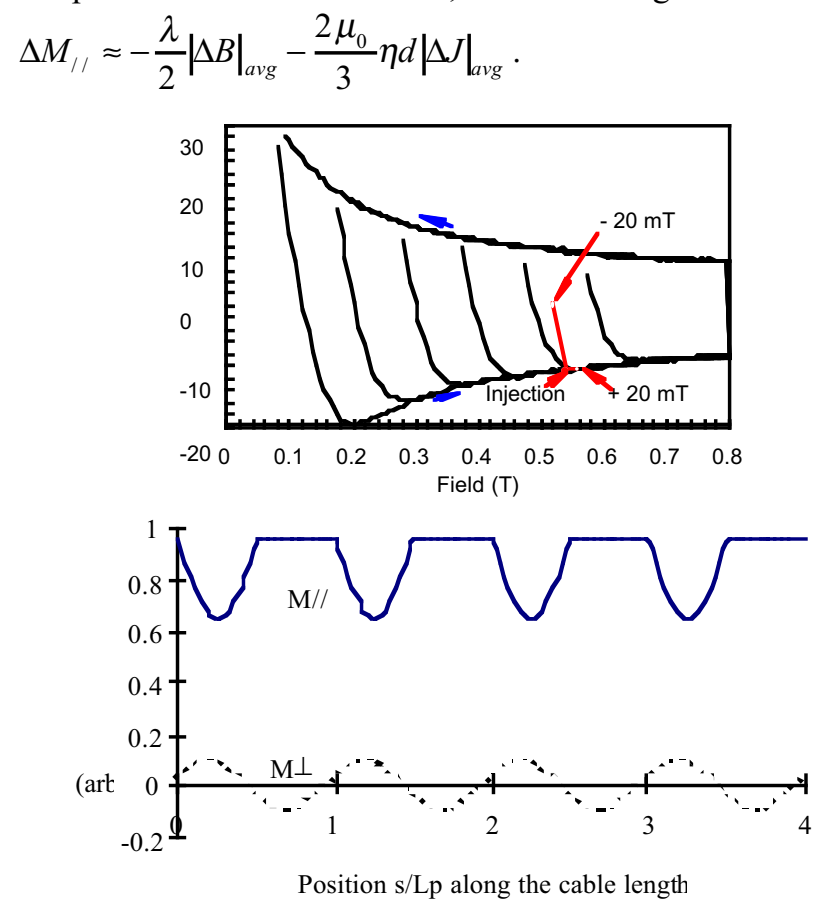

Fig.6. Magnetization change for a given field change of $\pm 20 \mathrm{mT}$ about the injection field (above) and decrease of the original magnetization $\mathrm{M} / /$ and the perpendicular magnetization $\mathrm{M} \perp$ for a periodic field change along the magnet (below). 
As soon as the magnet is ramped this effect is cancelled by the large background field change. We have estimated in Table II the changes $|\Delta J|_{\text {avg }}$ and $|\Delta B|_{\text {avg }}$ that are necessary to explain the observed decays of the field harmonics. Comparing the $|\Delta J|_{\text {avg }}$ to the typical transport current density at injection ( 2 orders of magnitude smaller) we conclude that the selffield effect is not by itself sufficient to explain the decay. The $|\Delta B|_{\text {avg }}$ required, on the other hand, is small. Such a field change would correspond to a change of the strand current of the order of $100 \mathrm{~A}$, and we recall that current imbalances of this order of magnitude have been observed the LHC dipoles[13]. Also note that the calculated $|\Delta \mathrm{B}|_{\text {avg }}$ of $12 \mathrm{mT}$ is of same order as the field change required for snap-back (15 to $20 \mathrm{mT}$, see also Fig. 3). Therefore the external field effect seems to be the dominating term.

According to (4) the decay and snap-back should not depend on temperature for changes of the magnetization which are small compared to the original magnetization of the strand. If however the temperature is changed simultaneously with the current redistribution process, the snap-back depends on the final temperature reached (see the results of Fig.4). Compared to constant temperature conditions, a temperature increase results in a smaller snap-back while a temperature decrease gives a larger one. The change in the snap-back - for a comparable absolute temperature change - is larger in the case of a temperature decrease. Finally, if the external field effect dominates and the field change is small, the decay and snap-back only depends on the internal field changes and is independent of the filament diameter.

\section{CONCLUSIONS}

We have explored several parameters influencing field decay and snap-back. We have shown experimentally that snapback is a DC magnetization recovery phenomenon. We propose that the mechanism causing the change of magnetization (and the field decay) during a constant current plateau is the change of the cable internal field resulting from current redistribution within the cable. The orders of magnitude estimated for this mechanism are consistent with measurements reported here and elsewhere.

From a practical standpoint, slow ramping after injection increases the snap-back duration, while a pre-injection stop at a current just below the injection level (by at least 20 to 30 A, the current level necessary to recover the field decay) decreases the snap-back amplitude.

TABLE II

Internal Field and Strand CurRent Changes Giving the Measured Field ERROR DECAY

\begin{tabular}{|c|c|c|}
\hline $\begin{array}{l}\text { Typical width of a strand magnetization hysteresis loop } \\
\text { at } B_{1}=0.5 \mathrm{~T} \text { and } \mathrm{T}=2 \mathrm{~K} \text { and } \mathrm{d}=5 \mu \mathrm{m} \text {. }\end{array}$ & (T) & \\
\hline $\begin{array}{l}\text { Typical critical current density of strand at } B_{1}=0.5 T \\
\text { and } T=2 K \text {. }\end{array}$ & & $7.0 \mathrm{E}+$ \\
\hline urrent density at injection for inner cable & & $2.4 \mathrm{E}+07$ \\
\hline he magnet) & & -0.0018 \\
\hline $\mathrm{Fig} 1$ & & \\
\hline effect only. & ( $\mathrm{T}$ & \\
\hline $\begin{array}{l}\text { equired }|\Delta \mathrm{J}|_{\text {avg }} \text { for } \mathrm{s} \\
\text { verage strand curr } \\
\text { lly }\end{array}$ & $(\mathrm{A} / \mathrm{n}$ & \\
\hline
\end{tabular}

We wil focus in the future on reproducibility issues and on the understanding of the current distribution process.

\section{REFERENCES}

[1] The Large Hadron Collider - Conceptual Design, CERN/AC/95-05, 1995.

[2] L. Bottura, A. Faus-Golfe, L. Walckiers, R. Wolf, "Field Quality of the Main Dipole Magnets for the LHC Accelerator", in press, 1996.

[3] D.A.Finley, et al., "Time Dependent Chromaticity Changes in the Tevatron", Proc. of $12^{\text {th }}$ Particle Accelerator Converence, Washington DC, March 16-19, 1987.

[4] D.A.Herrup, et al., "Time Variations of Fields in Superconducting Magnets and their Effects on Accelerators", IEEE Trans. Magn., 25, (2), 1643-1646, 1989.

[5] R.W. Hanft, et al., "Studies of Time Dependent Fields in Tevatron Superconducting Dipole Magnets", IEEE Trans. Magn., 25, (2), $1647-1651,1989$

[6] A. Devred, et al., "Time Decay Measurements of the Sextupole Component of the Magnetic Field in a 4-cm Aperture, 17-m-Long SSC Dipole Magnet Prototype", Proc. of $14^{\text {th }}$ Particle Accelerator Converence, San Francisco CA, May 6-9, 1991.

[7] H. Brueck, et al., "Time Dependent Field Distorsions from Magnetization Currents in the Superconducting Hera Magnets", Cryogenics, 30, 605-609, 1990.

[8] H. Brück et al., "Observation of a periodic pattern in the persistent current fields of the superconducting HERA magnets", Proc. of $14^{\text {th }}$ Particle Accelerator Converence, San Francisco CA, May 6-9, 1991.

[9] A.K. Ghosh, K.E. Robins, W.B. Sampson, "Axial Variations in the Magnetic Field of Superconducting Dipoles", Supercollider 4, 765772,1992

[10] J. Buckley, et al., "Dynamic Magnetic Measurements of Superconducting Magnets for LHC", IEEE Trans. Appl. Sup., 5 (2), 1024 $1027,1995$.

[11] R. Stiening, "A Possible Mechanism for Enhanced Persistent Current Sextupole Decay in SSC Dipoles", SSCL-359, 1991, unpublished

[12] M. Wilson, Superconducting Magnets, Clarendon Press, Oxford, 1983

[13] L. Bottura, L. Walckiers, Z. Ang, Experimental Evidence of Boundary Induced Coupling Currents in LHC Prototypes, Presented at 1996 Appl. Sup. Conf, Pittsburgh, unpublished. 